Available online on 15.02 .2019 at http://jddtonline.info
Open Access to Pharmaceutical and Medical Research

Open $\odot$ Access

Mini Review

\title{
Role of Inhibitors in Controlling the Diseases
}

Xiaochai Zhu

School of Pharmacy, Nanjing University of Chinese Medicine, Nanjing, Jiangsu, PR China, 210023

\begin{abstract}
Inhibitors are those particular molecules that have unique properties to control the function of a disease-causing agent. The review sheet discusses the list of inhibitors for every disease and the ideal mechanism to excite the same for a better application and impact. Most of the inhibitors in this report are noncompetitive which means that they can survive and give an efficient result to the disease on its own without the need for an external inhibitor.
\end{abstract}

Keywords: Inhibitors, disease-causing agent, enzymes activity

Article Info: Received 21 Dec 2018; $\quad$ Review Completed 25 Jan 2019; $\quad$ Accepted 28 Jan 2019; Available online 15 Feb 2019

\section{Cite this article as:}

Zhu X, Role of Inhibitors in Controlling the Diseases , Journal of Drug Delivery and Therapeutics. 2019; 9(1-s):381-383 http://dx.doi.org/10.22270/jddt.v9i1-s.2269

\section{*Address for Correspondence:}

Xiaochai Zhu, School of Pharmacy, Nanjing University of Chinese Medicine, Nanjing, Jiangsu, PR China, 210023

\section{Introduction}

Inhibitors are said to be molecules that establish links with enzymes and block its activity. This is ideal for dealing with metabolic imbalance ${ }^{1-4}$. Inhibitors can be competitive, uncompetitive and mixed. Every inhibitor can cure different diseases. The purpose of this review report is to highlight three major inhibitors to cure cancer, Alzheimer's disease and neuro disorders respectively. The identified inhibitors are found to bind with the agents that cause the disease to the patient's body.

\section{Inhibitors for cancer}

The biochemical process implies the interaction between proteins. Hypoxia is a condition that can be seen in cases involving stroke and chronic kidney failure. Peptidic inhibitors tend to perform better regarding inhibition and stabilization with the help of the pVHL/HIF-1 $\alpha$ inhibitor ${ }^{5-8}$. There are different docking structures followed to increase efficiency in the repression. The amide group in this inhibitor performs two actions - hydrogen bond donor as well as acceptor ${ }^{9-13}$.

The docking program is the key to efficient inhibition. Crossdocking helps to create a healthy crystal structure that is ideal for the potential inhibitor to act on it and control the functions of the disease-causing agent. The inhibitory activity happens against the pVHL/HIF-1 $\alpha$ interaction between proteins thereby resulting in better binding 5 .8 There is a need to work on the molecular optimization so that minimal compounds can assure higher potency and also serve as a promising lead compound to control cancer. This discovery of new inhibitors can be allowed to interact utilizing cascade docking and shape based screening methods.

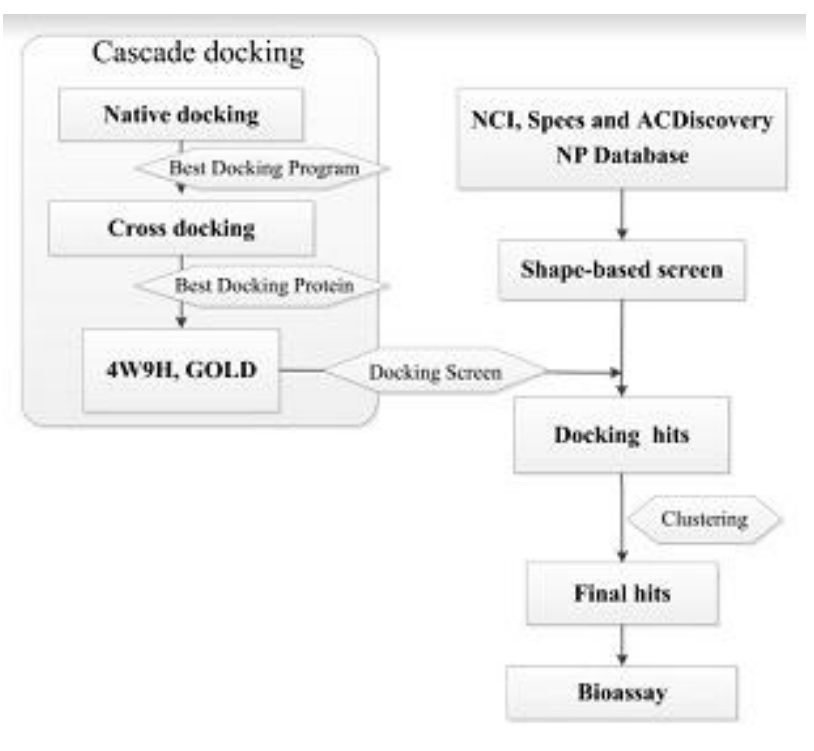




\section{Inhibitors for Alzheimer's disease}

Alzheimer's disease is a progressive neurological disorder that has multiple side effects. So far, there are just two inhibitors used namely acetylcholinesterase inhibitor and $\mathrm{N}$ methyl-D-aspartate receptor ${ }^{14-19}$. The multi-target directed ligands tend to contain multi-functional acetylcholinesterase inhibitors (AChEIs). After a shape-based virtual screening test, it has been observed that T5369186 is a cholinesterase inhibitor that can be used to treat cancer and other degenerative diseases ${ }^{6}$. To see the effectiveness of AChE, in vitro test followed by molecular docking is performed.

Molecular docking method is used to observe the relationships and activities between two or more compounds $5-8,20-24$. The amino chains are assessed here for their interactions. During the test followed by analysis, it was observed that 4-aminoquinoline is the core to create new cholinesterase inhibitors. This inhibitor is already in use to treat Alzheimer's disease. This can be altered with the movement of quinolone to different positions to check the specific location/ core that can create multifunctional AChEIs 6 .

The use of AChEI helps to develop the inhibitory potency, and the molecular design is well structured. Irrespective of the compounds present, AChEI can penetrate deeper and bind with other nano-molecules. Additionally, this inhibitor can to control the inflammatory condition associated with the nervous system and offers better relief thereby creating a normal situation in the affected area. Novel inhibitors are required shortly to control similar such progressive disorders $25-32$.

\section{Inhibitors for melanoma}

To treat skin pigmentation and melanoma, targeting of tyrosinase is performed so far. Melanin is a continuous production process that damages the skin due to exposure to radiation. Tyrosinase acts as an enzyme meant to control melanogenesis. With the widespread presence of melanoma, it is seen that there is an overexpression of tyrosinase ${ }^{33-40}$. This has led to the growth of dopamine which in turn produces dopamine quinone and dopaquinone ${ }^{5}$. Still, there is a need to identify new tyrosinase inhibitors.

For this process, shape-based overlays are used. The rapid overlays align between the molecules and have unique features including hydrophobes, rings, hydrogen bond acceptor and donor. The best way to determine the novel inhibitors for melanoma is to perform virtual screening. This is a natural process, but there are drawbacks as well such as poor availability, poor activity, and selectivity. The virtual screening also determines novel inhibitors that have lower cytotoxicity. The identified tyrosinase inhibitor indexed as 5186-0429 is found to eliminate inflammation and improve the resistance with its kinetic and molecular properties. This inhibitor can act as a quick heal to the case of melanoma ${ }^{25-29}$.

\section{Inhibitors for heart disease}

Another inhibitor/ mediator to protect the cardiac activity of a person is protein kinase C. It is already known that adenosine helps in cardioprotection. To know more about this inhibitor, the signaling mechanism is used, and this mechanism also helps to reveal the properties involved in the control of mitochondria ${ }^{10}$. Adenosine receptor activation can help to translocate protein kinase $\mathrm{C}$ isoforms and the latter will in turn bind with caveolin scaffolding. This can eliminate the blockages present in the pathway ${ }^{10}$.

As cardioprotection is influenced by age factors, the success of mitochondrial translocation is still unknown. There is a need for a natural inhibitor that can induce adenosine and target the right PKC isoforms that can protect from ischemic injury and other cardiac diseases.

\section{Conclusion}

Every inhibitor that is seen in today's health environment has multiple functions. Such inhibitors stand as the foundation to control many diseases including heart, melanoma, Alzheimer's and cancer. This paper has produced a list of inhibitors and the method to discover novel inhibitors that can help achieve a better cure to the disease ${ }^{5-}$ 8. The excitation, screening, docking, and application vary from one inhibitor to the other, but all the inhibitors above are safe to use to treat the patients and also expect long-term results.

\section{References}

1. Sun, Y. \& Kang, C. Self-Assembly of Peptides into Hydrogel. Journal of Organic \& Inorganic Chemistry 2016; 2:5.

2. Yao, Z., Sun, Y. \& Kang, C. Structure and self-assembly of multicolored Naphthalene Diimides Semiconductor. Nano LIFE 2016; 6:1642007.

3. Cheng, X., et al. T7 Peptide-Conjugated Lipid Nanoparticles for Dual Modulation of Bcl-2 and Akt-1 in Lung and Cervical Carcinomas. Molecular pharmaceutics 2018; 15:4722-4732.

4. Zhong, X., Sun, Y., Kang, C. \& Wan, G. The theory of dielectrophoresis and its applications on medical and materials research. European Journal of BioMedical Research 2017; 2:7-11.

5. Li, Q., et al. Identification by shape-based virtual screening and evaluation of new tyrosinase inhibitors. PeerJ 2018; 6:e4206.

6. Chen, Y., et al. Identification of 4-aminoquinoline core for the design of new cholinesterase inhibitors. PeerJ 2016; 4:e2140.

7. Kang, C. \& Hu, K. Impact of hypoxia in the expression and regulation of the TASK-1 potassium channel in cardiac myocytes. The FASEB Journal 2016; 30:lb598-lb598.

8. Kang, C. Ion channels, protein kinase $C$ and caveolae in cardioprotection, (The Ohio State University, 2015).

9. Qiao, H., et al. Redox-triggered mitoxantrone prodrug micelles for overcoming multidrug-resistant breast cancer. Journal of drug targeting 2018; 26:75-85.

10. Kang, C., Qin, J., Osei, W. \& Hu, K. Regulation of protein kinase Cepsilon and its age-dependence. Biochemical and Biophysical Research Communications 2017; 482:1201-1206.

11. Sun, Y., et al. RGD Peptide-Based Target Drug Delivery of Doxorubicin Nanomedicine. Drug development research 2017; 78:283-291.

12. Kang, C. \& Hu, K. Role of caveolin-3 in adenosine-induced increase in mitochondrial PKCE. The FASEB Journal 2013; 27:1191.1197-1191.1197.

13. Cheng, X. \& Lee, R.J. The role of helper lipids in lipid nanoparticles (LNPs) designed for oligonucleotide delivery. Adv Drug Deliv Rev 2016; 99: 129-137.

14. Davis, M.E., Chen, Z.G. \& Shin, D.M. Nanoparticle therapeutics: an emerging treatment modality for cancer. Nat Rev Drug Discov 2008; 7:771-782.

15. Kang, C., Sun, Y., Wang, M. \& Cheng, X. Nanosized camptothecin conjugates for single and combined drug delivery. European Journal of BioMedical Research 2016; 2:8-14.

16. Qiao, H., et al. Orally delivered polycurcumin responsive to bacterial reduction for targeted therapy of inflammatory bowel disease. Drug Delivery 2017; 24:233-242.

17. Liu, F., Sun, Y., Kang, C. \& Zhu, H. Pegylated Drug Delivery Systems: From Design to Biomedical Applications. Nano LIFE 2016; 6:1642002.

18. Sun, Y., Kang, C., Yao, Z., Liu, F. \& Zhou, Y. Peptide-Based Ligand for Active Delivery of Liposomal Doxorubicin. Nano Life 2016; 6:1642004.

19. Yeh, C.Y., Hsiao, J.K., Wang, Y.P., Lan, C.H. \& Wu, H.C. Peptideconjugated nanoparticles for targeted imaging and therapy of prostate cancer. Biomaterials 2016; 99:1-15.

20. Waller, A.P., et al. GLUT12 functions as a basal and insulinindependent glucose transporter in the heart. Biochimica et Biophysica Acta (BBA)-Molecular Basis of Disease 2013; 1832:121-127.

21. Yung, B.C., et al. Lipid nanoparticles composed of quaternary amine-tertiary amine cationic lipid combination (QTsome) for 
therapeutic delivery of AntimiR-21 for lung cancer. Molecular pharmaceutics 2016; 13:653-662.

22. Cheng, X., et al. Lipid Nanoparticles Loaded with an Antisense Oligonucleotide Gapmer Against Bcl-2 for Treatment of Lung Cancer. Pharmaceutical research 2017; 34:310-320.

23. Fan, S. \& Chi, W. Methods for genome-wide DNA methylation analysis in human cancer. Brief Funct Genomics 2016; 15:432442.

24. Kang, C. \& Hu, K. Modulation of the two-pore domain potassium channel TASK-1 by caveolin-3. The FASEB Journal 2015; 29:845.814.

25. Sun, Y., Kang, C., Liu, F. \& Song, L. Delivery of antipsychotics with nanoparticles. Drug Development Research 2016; 77:393-399.

26. Kang, C., et al. Delivery of nanoparticles for treatment of brain tumor. Current Drug Metabolism 2016; 17:745-754.

27. Xue, X., et al. Discovery of novel inhibitors disrupting HIF$1 \alpha /$ von Hippel-Lindau interaction through shape-based screening and cascade docking. PeerJ 2016; 4:e2757.

28. Hersch, S.J., et al. Divergent protein motifs direct elongation factor P-mediated translational regulation in Salmonella enterica and Escherichia coli. MBio 2013; 4:e0180-00113.

29. Shuhong, X., et al. Dynamic expression of AQP4 in early stageof ischemia/reperfusion rats and cerebral edema. Chinese Pharmacological Bulletin 2016; 32:1433-1441.

30. Peng, J., et al. Enhanced Liver Regeneration After Partial Hepatectomy in Sterol Regulatory Element-Binding Protein (SREBP)-1c-Null Mice is Associated with Increased Hepatocellular Cholesterol Availability. Cellular Physiology and Biochemistry 2018; 47:784-799.

31. Yang, Z., et al. Functional exosome-mimic for delivery of siRNA to cancer: in vitro and in vivo evaluation. Journal of Controlled Release 2016; 243:160-171.
32. Kang, C., Hernandez, V.A. \& Hu, K. Functional interaction of the two-pore domain potassium channel TASK-1 and caveolin-3. Biochimica et Biophysica Acta (BBA)-Molecular Cell Research 2017; 1864:1537-1544.

33. Kang, C., Qin, J., Osei, W. \& Hu, K. Age-dependent Mitochondrial Targeting Of Protein Kinase C Epsilon In Cardioprotection. The FASEB Journal (2017).

34. Han, R., Sun, Y., Kang, C., Sun, H. \& Wei, W. Amphiphilic dendritic nanomicelle-mediated co-delivery of 5-fluorouracil and doxorubicin for enhanced therapeutic efficacy. Journal of Drug Targeting 2017; 25:140-148.

35. Duan, Y., et al. Bioactivity evaluation-based ultra highperformance liquid chromatography coupled with electrospray ionization tandem quadrupole-time-of-flight mass spectrometry and novel distinction of multi-subchemome compatibility recognition strategy with Astragali Radix-Fructus Corni herbpair as a case study. J Pharm Biomed Anal 2016; 129:514-534.

36. Sun, Y., et al. Co-delivery of dual-drugs with nanoparticle to overcome multidrug resistance. European Journal of BioMedical Research 2016; 2: 12-18.

37. Ai, R., et al. Comprehensive epigenetic landscape of rheumatoid arthritis fibroblast-like synoviocytes. Nat Commun 2018; 9:1921.

38. Fan, S., et al. Computationally expanding infinium HumanMethylation450 BeadChip array data to reveal distinct DNA methylation patterns of rheumatoid arthritis. Bioinformatics 2016; 32:1773-1778.

39. Liu, F., Sun, Y. \& Kang, C. Controlling Amphiphilic Functional Block Copolymers' Self-Assembly: From Structure to Size. (2016).

40. Song, L., et al. Crocetin inhibits lipopolysaccharide-induced inflammatory response in human umbilical vein endothelial cells. Cellular Physiology and Biochemistry 2016; 40:443-452. 